\title{
Relatively complete ordered fields without integer parts
}

by

\author{
Mojtaba Moniri and Jafar S. Eivazloo (Tehran)
}

\begin{abstract}
We prove a convenient equivalent criterion for monotone completeness of ordered fields of generalized power series $\left[\left[F^{G}\right]\right]$ with exponents in a totally ordered Abelian group $G$ and coefficients in an ordered field $F$. This enables us to provide examples of such fields (monotone complete or otherwise) with or without integer parts, i.e. discrete subrings approximating each element within 1 . We include a new and more straightforward proof that $\left[\left[F^{G}\right]\right]$ is always Scott complete. In contrast, the Puiseux series field with coefficients in $F$ always has proper dense field extensions.
\end{abstract}

1. Introduction and preliminaries. A subset $C$ of an ordered field $F$ is a cut if $C<F \backslash C$. A nontrivial cut is a gap whenever it fails to have a least upper bound in the field. A gap $G$ in $F$ is regular whenever $\left(\forall \varepsilon \in F^{>0}\right)(G+\varepsilon \nsubseteq G)$. An ordered field $F$ is Scott complete if it does not have any proper extensions to an ordered field in which it is dense, equivalently it does not have any regular gaps, equivalently all Cauchy nets in the field of length equal to its cofinality converge there. It was proved in [12, Thm. 1] that any ordered field $F$ has a (unique up to an isomorphism of ordered fields which is identity on $F$ ) Scott completion. It is characterized by being Scott complete and having $F$ dense in it.

Monotone complete ordered fields were introduced in [6]. They are ordered fields with no bounded strictly increasing divergent functions. In such ordered fields, those nontrivial cuts which do not have a least upper bound in the field, are also not traversed by strictly increasing nets of length equal to the cofinality of the field. As implied by [11, Cor. 2.7], there are monotone complete ordered fields of any uncountable cofinality and so there exist plenty of monotone complete ordered fields not isomorphic to $\mathbb{R}$. On the other hand, it is clear that there are no monotone complete ordered fields of countable cofinality except (those isomorphic to) $\mathbb{R}$.

2000 Mathematics Subject Classification: 12J15, 13J05, 54H13.

Key words and phrases: ordered fields, Scott complete, monotone complete, generalized power series, integer part. 
A discrete subring of an ordered ring with 1 is said to be an integer part (IP) there if it approximates any element of the ring within 1 (equivalently within a finite distance). There are ordered fields with no IP's (see [1]). On the other hand, it was proved in [9] that every real closed field has an IP. Our goal here is to show that there are Scott complete and even monotone complete ordered fields with no IP's. We will be dealing with ordered fields of generalized power series. We will show that they are always Scott complete and prove a convenient monotone completeness criterion for them.

For an ordered field $F$ and ordered Abelian group $G$, recall that the ordered field of generalized power series with exponents in $G$ and coefficients in $F$ is

$$
\left[\left[F^{G}\right]\right]=\left\{\sum_{g \in G} a_{g} t^{g} \mid \text { the support }\left\{g \mid a_{g} \neq 0\right\} \text { of } \sum_{g \in G} a_{g} t^{g} \text { is well ordered }\right\} .
$$

It is equipped with the natural addition, multiplication, and order defined by setting $\sum_{g \in G} a_{g} t^{g}>0$ if $a_{g_{0}}>0$, with $g_{0}$ the minimum of $\operatorname{Supp}\left(\sum_{g \in G} a_{g} t^{g}\right)$.

A well known necessary and sufficient condition for $\left[\left[F^{G}\right]\right]$ to be real closed is that $G$ be divisible and $F$ be real closed (see [10,6.10]).

Observe that $\operatorname{cf}\left(\left[\left[F^{G}\right]\right]\right)=\operatorname{cf}(G)$ (where $\operatorname{cf}$ denotes cofinality).

The field of Puiseux series with ascending exponents over an ordered field $F$ is defined to be $P_{F}=\bigcup_{n \geq 1}\left[\left[F^{\left(\frac{1}{n} \mathbb{Z}\right)}\right]\right]$. Moreover $P_{F}$ is real closed if and only if $F$ is.

Before getting to the main concern mentioned above regarding being relatively complete versus having integer parts for ordered fields, we show in the next section that all Scott complete and certain other ordered fields always have a proper dense subfield. Fraction fields of integer parts are dense subfields, but they need not be proper $\left(^{1}\right)$.

\section{Proper dense subfields in Scott complete and Puiseux ordered fields}

Proposition 2.1. Every Scott complete ordered field can be obtained by Scott completion of a proper (dense) subfield.

Proof. We first observe the following:

Claim. Every Scott complete ordered field $F$ is uncountable.

Proof. Let $F$ be a Scott complete ordered field. If $F$ is Archimedean, then it is isomorphic to $\mathbb{R}$. So assume that $F$ is non-Archimedean and of count-

$\left({ }^{1}\right)$ For an example, see (3.5) in L. van den Dries, Some model theory and number theory for models of weak systems of arithmetic, in: L. Pacholski et al. (eds.), Model Theory of Algebra and Arithmetic, Lecture Notes in Math. 834, Springer, Berlin, 1980, 346-362. On the other hand, when the fraction field of an IP is indeed proper in a real closed field, this fact may not be that trivial; see, e.g., [8, Theorem 3.4(ii)]. 
able cofinality. If there exists a coinitial sequence $\left(\alpha_{i}\right)_{i \in \mathbb{N}}$ in $F^{>0}$ such that $(\forall i)(\forall n)\left(\alpha_{i+1}<\alpha_{i}^{n}\right)$, then the assignment mapping any sequence $\left(r_{i}\right)_{i \in \mathbb{N}}$ in $\mathbb{Q}$ to the limit of the Cauchy sequence $\left(\sum_{i \leq n} r_{i} \alpha_{i}\right)_{n \geq 0}$ in $F$ is one-to-one. Otherwise, there exists an element $\alpha \in F^{>0}$ whose positive integer powers are coinitial in $F^{>0}$. Then the assignment mapping any sequence $\left(r_{i}\right)_{i \in \mathbb{N}}$ in $\mathbb{Q}$ to the limit of the Cauchy sequence $\left(\sum_{i \leq n} r_{i} \alpha^{i}\right)_{n \geq 0}$ in $F$ is one-to-one. Therefore $F$ is uncountable, establishing the Claim.

As shown in [3, Lemma 2.3], any uncountable ordered field has a dense transcendence basis over the rationals. The subfield generated by the rationals and all but one element of the transcendence basis is a proper dense subfield.

Below we give a class of ordered fields still with proper dense subfields, but this time also with proper dense field extensions.

Proposition 2.2. Let $F$ be an ordered field. Then the Puiseux series field $P_{F}$ over $F$

(i) has proper dense subfields,

(ii) is not Scott complete.

Proof. (i) Observe that $P_{F}$ contains $\left[\left[F^{\mathbb{Z}}\right]\right]$ and the latter is of cardinality $|F|^{\aleph_{0}}>\aleph_{0}$. So the above-mentioned argument of [3] applies again.

(ii) Let $t$ be the positive $F$-infinitely small element used to present the elements of $P_{F}$. The sequence $\left(\sum_{i \leq n} t^{\left(p_{i}^{2}+1\right) / p_{i}}\right)_{n \in \mathbb{N}}$, where $p_{i}$ is the $i$ th prime, is a Cauchy sequence in $P_{F}$ which diverges there. This divergence is due to the fact that the least common multiple of the denominators of the exponents of the terms forming elements of the sequence tends to infinity with $n$. The sequence is Cauchy since the exponents of the leading terms of the far enough tails of the sequence tend to infinity, and such powers of $t$ are coinitial in the positive part of the field.

3. Scott complete ordered fields with no integer parts. It was proved by Kaplansky in [5, Thm. 4] that pseudo-completeness (a notion stronger than Scott completeness) for valued fields is equivalent to their maximality. Previously, Krull had already proved that generalized power series fields with their natural valuation are maximal. So these fields are pseudo-complete and in particular Scott complete.

Some weaker versions of this fact were later proved again by simpler methods. It was shown in [7] that Laurent series ordered fields over any ordered fields are Scott complete. On the other hand, it was proved in $[2$, Thm. 1.33] that the ordered Abelian group of generalized power series with coefficients in $\mathbb{R}$ and exponents in an arbitrary totally ordered set is Scott complete. For an ordered group $G$ and ordered field $F$, we showed in the 
proceedings paper [8] that if $F$ is Scott complete, then so is $\left[\left[F^{G}\right]\right]$. For convenience, we bring the proof here:

Proposition 3.1 (a stronger result to follow). For any ordered abelian group $G$ and ordered field $F$, the generalized power series field $\left[\left[F^{G}\right]\right]$ is Scott complete if so is $F$.

Proof. Let $\lambda$ be the cofinality of $G$ and consider a strictly decreasing $\lambda$ net $\left(a_{\theta}\right)_{\theta<\lambda}$ in $G^{<0}$ which is unbounded below there. Assume $\mathcal{F}:\left[\left[F^{G}\right]\right] \rightarrow$ $\left[\left[F^{G}\right]\right]$ is Cauchy. For each $g \in G$, define $f_{g}: \lambda \rightarrow F$ by

$$
f_{g}(\theta)=\left(\mathcal{F}\left(\chi_{\left\{a_{\theta}\right\}}\right)\right)(g) \text { for } \theta<\lambda .
$$

Claim 1. For all $g \in G$, the net $\left(f_{g}(\theta)\right)_{\theta<\lambda}$ is convergent in $F$.

Proof. Fix $g \in G$ and $\varepsilon \in F^{>0}$. By the Cauchy condition for $\mathcal{F}$, there exists $\theta_{0}<\lambda$ such that for all $\alpha, \beta \in\left[\left[F^{G}\right]\right]$ with $\alpha, \beta \geq \chi_{\left\{a_{\theta_{0}}\right\}}$, we have $|\mathcal{F}(\alpha)-\mathcal{F}(\beta)|<\varepsilon \chi_{\{g\}}$. This shows that $|\mathcal{F}(\alpha)(g)-\mathcal{F}(\beta)(g)|<\varepsilon$. Therefore for all $\theta_{1}, \theta_{2} \geq \theta_{0}$,

$$
\left|f_{g}\left(\theta_{1}\right)-f_{g}\left(\theta_{2}\right)\right|=\left|\left(\mathcal{F}\left(\chi_{\left\{a_{\theta_{1}}\right\}}\right)\right)(g)-\left(\mathcal{F}\left(\chi_{\left\{a_{\theta_{2}}\right\}}\right)\right)(g)\right|<\varepsilon,
$$

since $\chi_{\left\{a_{\theta_{1}}\right\}}, \chi_{\left\{a_{\theta_{2}}\right\}} \geq \chi_{\left\{a_{\theta_{0}}\right\}}$. Hence the net $\left(f_{g}(\theta)\right)_{\theta<\lambda}$ is Cauchy in $F$ and so convergent there, since $F$ is Scott complete.

Let $\gamma: G \rightarrow F$ be defined by $\gamma(g)=\lim \left(f_{g}(\theta)\right)_{\theta<\lambda}$. $\gamma(g))$.

Claim 2. $(\forall \eta<\lambda)\left(\exists \theta_{0}<\lambda\right)\left(\forall \theta \geq \theta_{0}\right)\left(\forall g \in G^{<-a_{\eta}}\right)\left(\mathcal{F}\left(\chi_{\left\{a_{\theta}\right\}}\right)(g)=\right.$

Proof. For any $\varepsilon=\chi_{\left\{-a_{\eta}\right\}}$ with $\eta<\lambda$, there exists $\theta_{0}<\lambda$ such that for all $\theta_{1}, \theta_{2}<\lambda$ with $\theta_{1}, \theta_{2} \geq \theta_{0}$, we have

$$
\left|\mathcal{F}\left(\chi_{\left\{a_{\theta_{1}}\right\}}\right)-\mathcal{F}\left(\chi_{\left\{a_{\theta_{2}}\right\}}\right)\right|<\chi_{\left\{-a_{\eta}\right\}} .
$$

This shows that for all $\theta_{1}, \theta_{2} \geq \theta_{0}$ and $g<-a_{\eta}$, we have

$$
\left(\mathcal{F}\left(\chi_{\left\{a_{\theta_{1}}\right\}}\right)\right)(g)=\left(\mathcal{F}\left(\chi_{\left\{a_{\theta_{2}}\right\}}\right)\right)(g)=\gamma(g) .
$$

Therefore, for all $\theta \geq \theta_{0}$ and $g \in G^{<-a_{\eta}}$, we have $\mathcal{F}\left(\chi_{\left\{a_{\theta}\right\}}\right)(g)=\gamma(g)$.

Claim 3. $\gamma \in\left[\left[F^{G}\right]\right]$.

Proof. It suffices to show that the support of $\gamma$ is well ordered. For all $g \in G$, there exists $\eta<\lambda$ such that $g<-a_{\eta}$. Let $\theta_{0}$ be as in Claim 2. As $g$ cannot be the initial term of any infinite strictly decreasing sequence in the support of $\mathcal{F}\left(\chi_{\left\{a_{\theta_{0}}\right\}}\right)$, Claim 2 shows that the same holds for the support of $\gamma$.

Claim 4. The function $\mathcal{F}$ on $\left[\left[F^{G}\right]\right]$ tends to $\gamma$ at infinity.

Proof. It is enough, by the Cauchy criterion for $\mathcal{F}$, to apply $\mathcal{F}$ on those $f$ 's in $\left[\left[F^{G}\right]\right]$ that are of the form $\chi_{\left\{a_{\theta}\right\}}$ for $\theta<\lambda$, and let $\theta$ tend to $\lambda$. The result is then immediate from Claim 2. 
The above claims give the result.

Improving upon this, here we prove Scott completeness of $\left[\left[F^{G}\right]\right]$ for arbitrary ordered fields by a more straightforward method compared to the one mentioned at the beginning of this section:

THEOREM 3.2. For every Abelian (totally) ordered nonzero group $H$ and every ordered field $F$, the generalized power series field $\left[\left[F^{H}\right]\right]$ is Scott complete.

Proof. Assume for a contradiction that $G$ is a regular gap in $\left[\left[F^{H}\right]\right]$. Let $K$ be the Scott completion of $F$. Let $G^{\prime}$ denote the downward closure of $G$ in $\left[\left[K^{H}\right]\right]$.

Claim 1. We still have $G^{\prime}$ regular in $\left[\left[K^{H}\right]\right]$.

Proof. Fix an arbitrary $\varepsilon \in\left[\left[K^{H}\right]\right]^{>0}$. By the cofinality of $\left[\left[F^{H}\right]\right]$ in $\left[\left[K^{H}\right]\right]$, there exists $\delta \in\left[\left[F^{H}\right]\right]^{>0}$ such that $\delta<\varepsilon$. Now since the gap $G$ is regular in $\left[\left[F^{H}\right]\right]$, there exist $x \in G \subseteq G^{\prime}$ and $y \in\left[\left[F^{H}\right]\right] \backslash G \subseteq\left[\left[K^{H}\right]\right] \backslash G^{\prime}$ such that $y-x<\delta<\varepsilon$. This takes care of Claim 1 .

By Proposition 3.1, [[K $\left.\left.K^{H}\right]\right]$ is Scott complete. So there exists $\alpha \in\left[\left[K^{H}\right]\right]$ such that $\alpha=\sup G^{\prime}$. We can write $\alpha=\beta+a t^{h_{0}}+\gamma$, where $h_{0}$ is the least exponent in $H$ such that $\alpha\left(h_{0}\right)=a \notin F$ (it exists since $G$ was assumed to be a gap in $\left[\left[F^{H}\right]\right]$ and so not all coefficients of terms of $\alpha$ could belong to $F$ ), and $\beta$ and $\gamma$ denote the higher and lower terms of $\alpha$ respectively. The contradiction we are looking for is obtained from:

Claim 2. If $h_{1}>h_{0}$, then for all $x \in G$ and $y \in\left[\left[F^{H}\right]\right] \backslash G$, we have $y-x \geq t^{h_{1}}$.

Proof. If $x \in G, y \in\left[\left[F^{H}\right]\right] \backslash G$, and $y-x<t^{h_{1}}$, then for all $h<h_{1}$ we have $x(h)=y(h)=\alpha(h)$. So $x\left(h_{0}\right)=y\left(h_{0}\right)=\alpha\left(h_{0}\right)=a$, which is a contradiction, since $x, y \in\left[\left[F^{H}\right]\right]$.

Proposition 3.3. For any ordered field $F$ and totally ordered Abelian group $G$, the generalized power series field $\left[\left[F^{G}\right]\right]$ has an integer part if and only if $F$ does.

Proof. If $I$ is an integer part of $F$, then $\left[\left[F^{G^{<0}}\right]\right]+I$ is an integer part for $\left[\left[F^{G}\right]\right]$.

In the other direction, assume $I_{0}$ is an integer part of $\left[\left[F^{G}\right]\right]$. Then $I_{1}=I_{0} \cap\left[\left[F^{G^{\geq 0}}\right]\right]$ is an integer part of the $\operatorname{ring}\left[\left[F^{G^{\geq 0}}\right]\right]$. Note that $\left[\left[F^{G^{>0}}\right]\right]$ is a maximal ideal of $\left[\left[F^{G^{\geq 0}}\right]\right]$ and the residue field $\left[\left[F^{G^{\geq 0}}\right]\right] /\left[\left[F^{G^{>0}}\right]\right]$ (equipped with the natural order induced from the one on $\left[\left[F^{G{ }^{\geq 0}}\right]\right]$ by just identifying $F$-infinitely close elements) is isomorphic to $F$. One can see that $I_{1} /\left[\left[F^{G^{>0}}\right]\right]$ (in which all residue classes are singletons) is an integer part of $\left[\left[F^{G^{\geq 0}}\right]\right] /\left[\left[F^{G^{>0}}\right]\right]$, which therefore has a counterpart in $F$. 
EXAMPLE 3.4. The Laurent series ordered field $\left[\left[\mathbb{Q}^{\mathbb{Z}}\right]\right]$ with rational coefficients is Scott complete and has an integer part.

By [12, Thm. 2], $F$ is dense in $\mathrm{RC}(F)$ if and only if its Scott completion is real closed. Therefore, no proper dense subfield of the ordered field in the next result is dense in its real closure (since $\mathbb{Z}$ is not divisible).

COROLlary 3.5. There exist Scott complete ordered fields without integer parts.

Proof. Let $B$ be the ordered field constructed in [1] which has no integer parts. Then $\left[\left[B^{\mathbb{Z}}\right]\right]$ is such an ordered field.

\section{A monotone completeness criterion}

THEOREM 4.1 (monotone completeness criterion for generalized power series). For every ordered field $F$ and nontrivial (totally) ordered Abelian group $G,\left[\left[F^{G}\right]\right]$ is monotone complete if and only if $G$ does not contain any bounded strictly monotone nets of length $\mathrm{cf}(G)$ and $F$ does not contain any strictly monotone nets of length $\mathrm{cf}(G)$ (no matter whether bounded or not).

Proof. Let $\operatorname{cf}(G)=\lambda$. Then, as mentioned before, $\operatorname{cf}\left(\left[\left[F^{G}\right]\right]\right)=\lambda$ as well.

"Only if". Assume for a contradiction that $\left(g_{i}\right)_{i<\lambda}$ is a bounded strictly decreasing net in $G$ and so $\left(t^{g_{i}}\right)_{i<\lambda}$ is a bounded strictly increasing net in $\left[\left[F^{G}\right]\right]$. Since $\left[\left[F^{G}\right]\right]$ is monotone complete, the latter must converge there. But this cannot happen since in generalized power series fields, terms of convergent nets have their leading terms (as well as other ones, something nonexisting in our case) fixed deep enough in the net.

Next suppose, again for a contradiction, that $\left(a_{i}\right)_{i<\lambda}$ is a strictly increasing net in $F$. Then as the strictly increasing net $\left(a_{i}\right)_{i<\lambda}$ is bounded in $\left[\left[F^{G}\right]\right]$ and by the monotone completeness assumption, $\lim _{i<\lambda}\left(a_{i}\right)$ would exist in $\left[\left[F^{G}\right]\right]$. But this cannot happen since no net of elements in $F$ converges in $\left[\left[F^{G}\right]\right]$ unless it is eventually stabilized (as the latter has $F$-infinitesimals).

"If". Suppose $\left(\sigma_{i}\right)_{i<\lambda}$ is a bounded strictly increasing net in $\left[\left[F^{G}\right]\right]$. We show that it converges there. For every $i<\lambda$, there exists an ordinal $\eta_{i} \leq \lambda$ such that the support of $\sigma_{i}$ equals $\left(g_{i, j}\right)_{j<\eta_{i}}$, a strictly increasing net which is bounded below, but not necessarily above. The reason that $\eta_{i} \leq \lambda$ for every $i$ is that otherwise the strictly increasing $\lambda$-net $\left(g_{i, j}\right)_{j<\lambda}$ would be bounded above by any of the chopped off tail terms, contradicting the assumption on $G$. Next we can assume, without loss of generality, that $\eta_{i}=\lambda$ for every $i<\lambda$. The reason is that we can add to each $\sigma_{i}$ an $F$-infinitesimal element of $\left[\left[F^{G}\right]\right]$ whose support is unbounded above in $G$ (obviously the original net converges if and only if the latter does).

For each $i, j<\lambda$, let $\sigma_{i}\left(g_{i, j}\right)=a_{i, j}$.

Claim. $(\forall j<\lambda)(\exists i<\lambda)(\forall I \geq i)(\forall l \leq j)\left(g_{I, l}=g_{i, l} \wedge a_{I, l}=a_{i, l}\right)$. 
Proof. We use induction on ordinals $\kappa<\lambda$. Suppose $\kappa<\lambda$ is such that $(\forall j<\kappa)(\exists i<\lambda)(\forall I \geq i)(\forall l \leq j)\left(g_{I, l}=g_{i, l} \wedge a_{I, l}=a_{i, l}\right)$. We show that there exists $i<\lambda$ such that for all $I \geq i$, we have $g_{I, \kappa}=g_{i, \kappa}$ and $a_{I, \kappa}=a_{i, \kappa}$. As $\lambda$ is a regular cardinal, there exists $\eta<\lambda$ such that $(\forall I \geq$ $\eta)(\forall l<\kappa)\left(g_{I, l}=g_{\eta, l} \wedge a_{I, l}=a_{\eta, l}\right)$. So the net $\left(g_{i, \kappa}\right)_{\eta \leq i<\lambda}$ is decreasing (since $\left(\sigma_{i}\right)_{\eta \leq i<\lambda}$ is increasing). It is also bounded below by, say, $g_{\eta, 0}$. Now if $\left(g_{i, \kappa}\right)_{\eta \leq i<\lambda}$ did not get stabilized, then a strictly decreasing subnet which is bounded below (of length $\lambda$, by regularity again) could be extracted from it, which would contradict the assumption on $G$. So there exists $\zeta<\lambda$, which we assume without loss of generality to be greater than $\eta$, such that $(\forall I \geq \zeta)\left(g_{I, \kappa}=g_{\zeta, \kappa}\right)$. This also shows that $\left(a_{I, \kappa}\right)_{\zeta \leq I<\lambda}$ is increasing. Now by the assumption on $F$, it must eventually stabilize (use a similar reasoning to that for the $g$ 's). So for some $i \geq \zeta$, we have $(\forall I \geq i)\left(a_{I, \kappa}=a_{i, \kappa}\right)$ (in addition to $\left.g_{I, \kappa}=g_{i, \kappa}\right)$. This finishes the Claim.

Let $\sigma=\sum_{j<\lambda} a_{i_{j}, j} t^{g_{i_{j}, j}}$, where for each $j<\lambda, i_{j}$ is a witness ordinal for the existential quantifier in the Claim above. Note that $\left(\forall j_{1}, j_{2}<\lambda\right)$ $\left(j_{1}<j_{2} \Rightarrow g_{i_{1}, j_{1}} \leq g_{i_{2}, j_{1}}<g_{i_{j_{2}}, j_{2}}\right)$ and so $\left(g_{i_{j}, j}\right)_{j<\lambda}$ is strictly increasing. Hence $\sigma \in\left[\left[F^{G}\right]\right]$. We show that $\lim _{i<\lambda} \sigma_{i}=\sigma$. By our assumption on $G$, the strictly increasing net $\left(g_{i_{j}, j}\right)_{j<\lambda}$ cannot be bounded above there. So for any given $\varepsilon=t^{g} \in\left[\left[F^{G}\right]\right]^{>0}$, there exists $j<\lambda$ such that $g_{i_{j}, j}>g$. For all $i>i_{j}$, by the Claim above, we have $\left|\sigma_{i}-\sigma\right|<t^{g_{i_{j}, j}}<\varepsilon$.

The two ordered fields above are not monotone complete as the coefficient fields $\mathbb{Q}$ and $B$ (like any other ordered field) have strictly increasing nets of length $\mathrm{cf}(\mathbb{Z})=\omega$. If we replace the ordered group of exponents there by a direct lexicographically ordered sum of continuum many copies of $\mathbb{Z}$, the resulting power series fields will still be monotone incomplete. In these cases, the group of exponents has bounded strictly increasing nets like $\left(g_{\lambda}\right)_{\lambda<c}$ with $g_{\lambda}(\alpha)=1$ if $\alpha<\lambda$ and $g_{\lambda}(\alpha)=0$ otherwise. We provide monotone complete $\left[\left[F^{G}\right]\right]$ 's with or without integer parts in our last section below.

5. Monotone complete ordered fields with no IP's. To get monotone complete ordered fields of generalized power series, we will still take the coefficient field of countable cofinality, namely $\mathbb{Q}$ and the Boughattas field $B$ (to have or not have an IP, respectively). Regarding the ordered group of exponents, we first recall the notion of an integer set in ordered fields, due to Keisler, which appeared in [11]. They are subsets uniquely approximating from below each element of the field within 1.

COROLlary 5.1. There exist monotone complete ordered fields without integer parts.

Proof. Once again, let $B$ be the ordered field constructed in [1] which has no integer parts. As mentioned before, [11, Cor. 2.7] shows that there 
are monotone complete ordered fields of any uncountable cofinality. Let $F$ be a monotone complete ordered field of an uncountable cofinality $\lambda$ large enough so that $B$ does not have any strictly increasing nets of length $\lambda$. It was shown in [11] that every ordered field has an additive subgroup which is an integer set for that field. Suppose that $G$ is an integer set and an additive subgroup of $F$. Note that $G$ does not contain any bounded strictly monotone nets of length $\lambda$. The reason is that since $F$ is monotone complete, such nets would converge in $F$ and so would be Cauchy in the discrete set $G$, which is impossible. We conclude that $\left[\left[B^{G}\right]\right]$ is monotone complete and has no integer parts.

Note that $\left[\left[\mathbb{Q}^{G}\right]\right]$ (with the same $G$ ) is also monotone complete (but this one does have an IP).

Alternatively, the hyper-real field $\mathbb{R}^{*}$ (see, e.g., [4] for an introduction) can be taken monotone complete; see [6]. The corresponding discretely ordered ring $\mathbb{Z}^{*}$ is an integer part for $\mathbb{R}^{*}$. Now consider $\mathbb{Z}^{*}$ as a totally ordered (additive) Abelian group. By the monotone completeness criterion, $\left[\left[\mathbb{Q}^{\mathbb{Z}^{*}}\right]\right]$ is monotone complete (and obviously has an integer part). Once again, let $B$ be the ordered field constructed in [1] which has no integer parts and is of countable cofinality. Then $\left[\left[B^{\mathbb{Z}^{*}}\right]\right]$ is monotone complete and has no integer parts.

Acknowledgements. The authors thank the Institute for Studies in Theoretical Physics and Mathematics (IPM), Tehran, Iran, for partial support. This work formed part of the second author's PhD thesis under the first author's supervision at Tarbiat Modarres University, Tehran, Iran, and was partially presented during Algebra Conference Venezia 2002, Venice, Italy, June 3-8, 2002.

\section{References}

[1] S. Boughattas, Résultats positifs et négatifs sur l'existence d'une partie entière dans les corps ordonnés, C. R. Acad. Sci. Paris Sér. I Math. 313 (1991), 701-704.

[2] H. G. Dales and W. H. Woodin, Super-Real Fields, Oxford Univ. Press, New York, 1996.

[3] P. Erdős, L. Gillman, and M. Henriksen, An isomorphism theorem for real-closed fields, Ann. of Math. 61 (1955), 542-554.

[4] R. Goldblatt, Lectures on the Hyperreals. An Introduction to Nonstandard Analysis, Springer, New York, 1998.

[5] I. Kaplansky, Maximal fields with valuations, Duke Math. J. 9 (1942), 303-321.

[6] H. J. Keisler, Monotone complete fields, in: Victoria Sympos. on Nonstandard Analysis, A. Hurd and P. Loeb (eds.), Lecture Notes in Math. 369, Springer, Berlin, 1974, $113-115$.

[7] D. Laugwitz, Bemerkungen über Stetigkeit und angeordnete Körper, Demonstratio Math. 6 (1973), 191-209. 
[8] M. Moniri and J. S. Eivazloo, Using nets in Dedekind, monotone, or Scott incomplete ordered fields and definability issues, in: Proc. Ninth Prague Topological Sympos. (2001), P. Simon (ed.), Topology Atlas (available electronically at http://at.yorku.ca), Toronto, 2002, 195-203.

[9] M.-H. Mourgues and J. P. Ressayre, Every real closed field has an integer part, J. Symbolic Logic 58 (1993), 641-647.

[10] P. Ribenboim, Fields: algebraically closed and others, Manuscripta Math. 75 (1992), $115-150$.

[11] J. H. Schmerl, Models of Peano arithmetic and a question of Sikorski on ordered fields, Israel J. Math. 50 (1985), 145-159.

[12] D. Scott, On completing ordered fields, in: Applications of Model Theory to Algebra, Analysis, and Probability, W. A. J. Luxemburg (ed.), Holt, Rinehart and Winston, New York, 1969, 274-278.

Mojtaba Moniri

Institute for Studies in Theoretical

Physics and Mathematics (IPM)

Tehran, Iran

E-mail: mojmon@ipm.ir

and

Department of Mathematics

Tarbiat Modarres University

Tehran, Iran

E-mail: moniri_m@modares.ac.ir
Jafar S. Eivazloo Institute for Studies in Theoretical Physics and Mathematics (IPM)

Tehran, Iran E-mail: eivazloo@ipm.ir

and

Department of Mathematics Tarbiat Modarres University Tehran, Iran

Received 28 May 2002;

in revised form 3 August 2003 